\title{
Biomechanics of transvertebral screw fixation in the thoracic spine: an in vitro study
}

\author{
Nestor G. Rodriguez-Martinez, MD, Amey Savardekar, MCh, Eric W. Nottmeier, MD, \\ Stephen Pirris, MD, Phillip M. Reyes, BSE, Anna G. U. S. Newcomb, MS, \\ George A. C. Mendes, MD, Samuel Kalb, MD, Nicholas Theodore, MD, and Neil R. Crawford, PhD
}

Department of Neurosurgery, Barrow Neurological Institute, St. Joseph's Hospital and Medical Center, Phoenix, Arizona

\begin{abstract}
OBJECTIVE Transvertebral screws provide stability in thoracic spinal fixation surgeries, with their use mainly limited to patients who require a pedicle screw salvage technique. However, the biomechanical impact of transvertebral screws alone, when they are inserted across 2 vertebral bodies, has not been studied. In this study, the authors assessed the stability offered by a transvertebral screw construct for posterior instrumentation and compared its biomechanical performance to that of standard bilateral pedicle screw and rod (PSR) fixation.
\end{abstract}

METHODS Fourteen fresh human cadaveric thoracic spine segments from T-6 to T-11 were divided into 2 groups with similar ages and bone quality. Group 1 received transvertebral screws across 2 levels without rods and subsequently with interconnecting bilateral rods at 3 levels (T8-10). Group 2 received bilateral PSR fixation and were sequentially tested with interconnecting rods at T7-8 and T9-10, at T8-9, and at T8-10. Flexibility tests were performed on intact and instrumented specimens in both groups. Presurgical and postsurgical 0 -arm 3D images were obtained to verify screw placement.

RESULTS The mean range of motion (ROM) per motion segment with transvertebral screws spanning 2 levels compared with the intact condition was $66 \%$ of the mean intact ROM during flexion-extension $(p=0.013), 69 \%$ during lateral bending $(p=0.015)$, and $47 \%$ during axial rotation $(p<0.001)$. The mean ROM per motion segment with PSR spanning 2 levels compared with the intact condition was $38 \%$ of the mean intact ROM during flexion-extension ( $p<0.001), 57 \%$ during lateral bending $(p=0.007)$, and $27 \%$ during axial rotation $(p<0.001)$. Adding bilateral rods to the 3 levels with transvertebral screws decreased the mean ROM per motion segment to $28 \%$ of intact ROM during flexion-extension $(p<0.001), 37 \%$ during lateral bending $(p<0.001)$, and $30 \%$ during axial rotation $(p<0.001)$. The mean ROM per motion segment for PSR spanning 3 levels was $21 \%$ of intact ROM during flexion-extension $(p<0.001), 33 \%$ during lateral bending $(p<0.001)$, and $22 \%$ during axial rotation $(p<0.001)$.

CONCLUSIONS Biomechanically, fixation with a novel technique in the thoracic spine involving transvertebral screws showed restoration of stability to well within the stability provided by PSR fixation.

http://thejns.org/doi/abs/10.3171/2015.11.SPINE15562

KEY WORDS transvertebral screws; posterior instrumentation; thoracic spine

I N 1994, Abdu et al. first described the technique of using transvertebral pedicle screws to fixate the lumbar spine. ${ }^{1}$ They performed L5-S1 fixation through the S-1 pedicle with good radiological and clinical outcomes. Other studies using the same technique have also shown good results. ${ }^{3,10}$ Transdiscal L5-S1 screw fixation for isthmic spondylolisthesis has been studied biomechanically, and results have not revealed any difference in stiffness between transdiscal fixation and combined interbody/pedicle screw fixation in unstable spines., ${ }^{2,11}$
In the thoracic spine, pedicle screw placement using the Roy-Camille method ${ }^{14}$ is one of the most widely used techniques for spinal screw fixation. In some cases, a partial laminectomy is a necessary adjuvant to pedicle screw placement in the thoracic spine.$^{15}$ The use of preoperative CT is helpful in taking the correct measurement of the pedicle width, and it gives clinicians a better idea about the hardware needed. ${ }^{4}$

The clinical use of transvertebral screws in the thoracic spine, based on the Abdu technique, ${ }^{1}$ was recently

ABBREVIATIONS LZ = lax zone; PSR = pedicle screw and rod; ROM = range of motion; $S Z$ = stiff zone; TTR = transvertebral screws with rods .

SUBMITTED May 11, 2015. ACCEPTED November 23, 2015.

INCLUDE WHEN CITING Published online April 1, 2016; DOI: 10.3171/2015.11.SPINE15562. 
described by Nottmeier and Pirris. ${ }^{12}$ These authors used 3D image guidance in patients with osteoporosis requiring a pedicle screw salvage technique. Their technique resulted in good radiological and clinical outcomes for these patients. However, no biomechanical studies have been performed to test the stability provided by this type of instrumentation.

The aim of this biomechanical study was to evaluate a transvertebral construct for posterior instrumentation and to compare its biomechanical performance with that of standard bilateral pedicle screw and rod (PSR) fixation in normal thoracic spines.

\section{Methods}

Fourteen fresh human cadaveric thoracic spine segments (T6-11) were used. The specimens were carefully cleaned of muscle tissue, while all ligaments, joint capsules, and discs were kept intact. Dual-energy x-ray absorptiometry scans were performed on the L- 4 vertebra of each specimen to assess bone mineral density. None of the specimens were osteoporotic. Plain film radiographs were taken and patient histories reviewed to ensure that no specimens had obvious flaws, metastatic disease, osteophytes, disc narrowing, or joint arthrosis. The specimens were divided into 2 groups (Fig. 1). Group 1 (4 males, 3 females; mean age at death, 48.2 years; age range, 26-59 years) received transvertebral screws and Group 2 (3 males, 4 females; mean age at death, 46.1 years; age range, 33-61 years) received PSR fixation. In preparation for the biomechanical tests, T-11 of each specimen was reinforced with household wood screws, embedded in a block of fastcuring resin (Smooth-Cast 300Q; Smooth-On, Inc.), and attached to the base of the testing apparatus. Similarly, the T-6 vertebra was also reinforced with household screws and embedded in resin in a cylindrical metal fixture for load application.

The flexibility of specimens in Group 1 was tested as follows: 1) intact; 2) after bilateral transvertebral screw fixation $(4.5 \times 50-\mathrm{mm}$ fixed-angle dual-threaded screws) spanning T7-8, T8-9, and T9-10 without rods (Fig. 1 left); and 3) after bilateral transvertebral screw fixation $(5 \times 50-$ $\mathrm{mm}$ polyaxial dual-threaded screws) at the same levels as in condition 2, with rods attached bilaterally and spanning T8-10 (i.e., transvertebral screws with rods [TTR]; Fig. 2). The screw size in testing step 3 of Group 1 was changed from that used in step 2 because polyaxial rather than fixed-head screws were necessary for rod attachment; removal and reinsertion of screws with the same diameter could have resulted in decreased fixation strength. The flexibility of specimens in Group 2 was tested as follows: 1) intact; 2$)$ with bilateral PSR fixation $(4.5 \times 50-\mathrm{mm}$ polyaxial dual-threaded screws) at T-7, T-8, T-9, and T-10 (Fig. 1 right) and rods spanning T7-8 and T9-10 (Fig. 3 left); 3 ) with bilateral PSR fixation $(4.5 \times 50-\mathrm{mm}$ polyaxial dualthreaded screws) and rods spanning T8-9 (Fig. 3 right); and 4) with bilateral PSR fixation $(5 \times 50-\mathrm{mm}$ polyaxial dual-threaded screws) spanning T8-10 (not shown). The screw size in testing step 3 of Group 2 was changed from that used in step 2 to allow us to make equivalent comparisons between constructs that had rods spanning the same

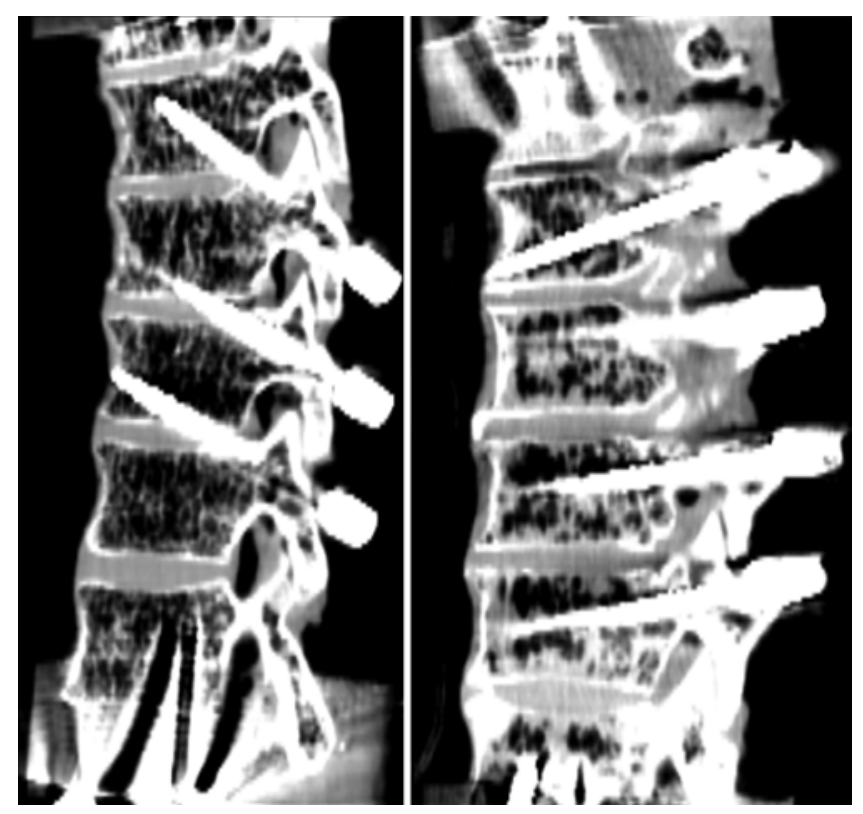

FIG. 1. Sagittal reconstructed image from the O-arm for postsurgical verification of screw placement with the 2 different constructs: Group 1, transvertebral screws (left) and Group 2, pedicle screws (right).

levels (T8-10) in Groups 1 and 2. The rod used was the same size (5.0-mm diameter) in all specimens and cases.

After localization of an anatomical entry point at the junction of the transverse process and lamina, screws in

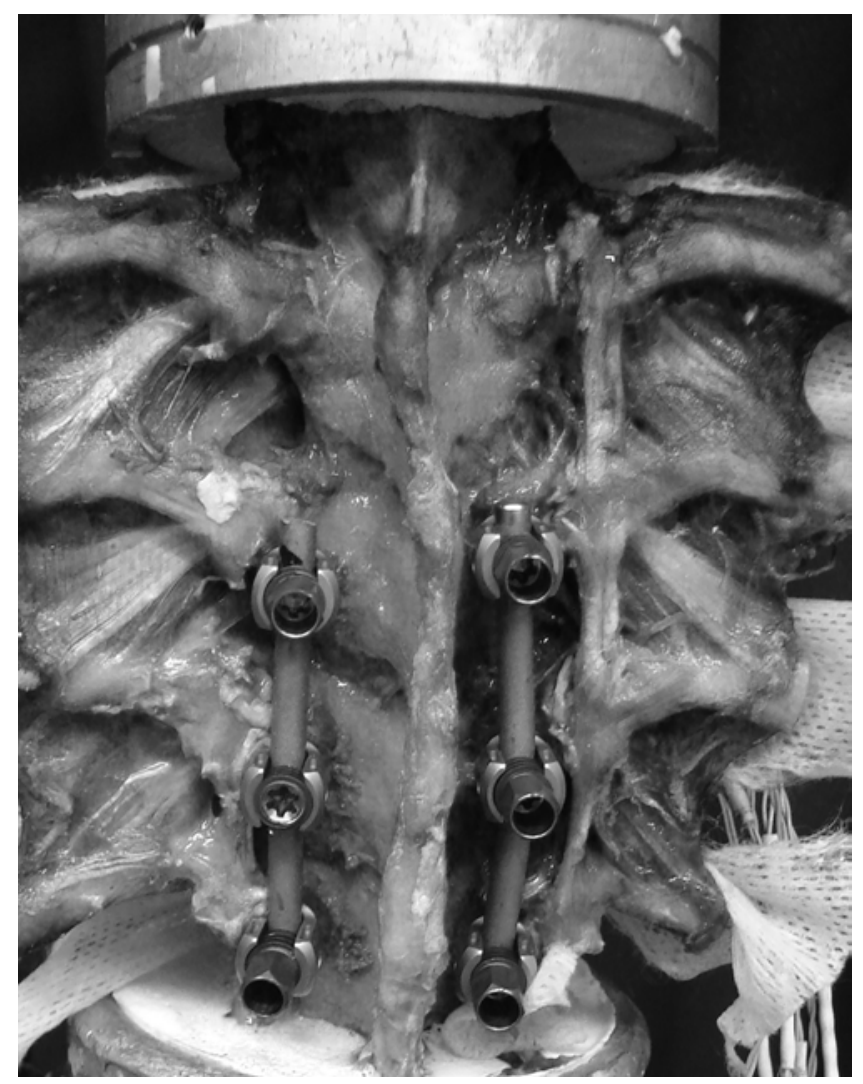

FIG. 2. Cadaveric specimen with rods attached to transvertebral screws spanning T8-10. 


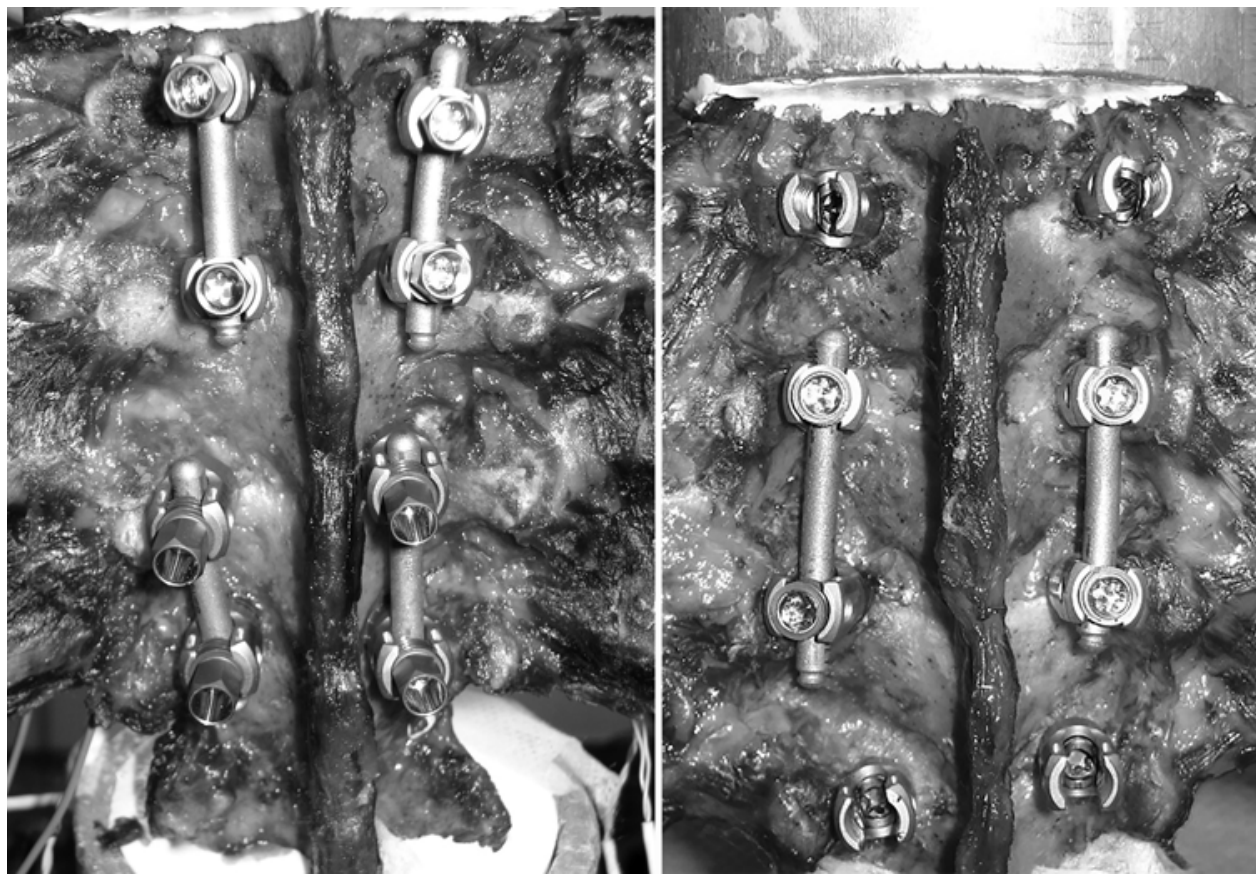

FIG. 3. Pedicle screw and rod fixation in cadaveric specimens with segmental rods attached at T7-8 and T9-10 (left) and with rods spanning T8-9 (right).

both groups were inserted at the lateral border of the pars. The position of the entry point was moved to a more medial and cephalad position on the posterior elements as one proceeds from the distal to the apical midthoracic region. All screws were inserted using the StealthStation navigation system (Medtronic Surgical Navigation Technologies), to ensure correct screw trajectories in all cases.

Presurgical and postsurgical 3D O-arm images (Medtronic Surgical Imaging Systems) were obtained in each case for screw size selection (length and diameter) and to verify screw placement (Fig. 1). Single-level PSR constructs were tested initially despite the presence of pedicle screws at 4 vertebral levels for a more equitable comparison between the groups. That is, specimens in Group 2 were sequentially tested first with interconnecting rods locking the T7-8 and T9-10 levels (Fig. 3 left), then with these rods removed and interconnecting rods locked at the T8-9 level (Fig. 3 right).

In all conditions for each specimen, an apparatus developed by Crawford et al. ${ }^{5}$ was used, in which a system of cables and pulleys impart nondestructive, nonconstraining torques in conjunction with a standard servo-hydraulic test system. With pure-moment loading, the same load is distributed to each level of the spine. This ensures an equivalent comparison between all levels regardless of the distance from the point of loading. ${ }^{13}$ Loads of $6.0 \mathrm{Nm}$ maximum were applied about the appropriate anatomical axes to induce bending or twisting in each plane of motion: flexion and extension, left and right lateral bending, and left and right axial rotation. In each loading direction, 3 preconditioning cycles were applied at $6.0 \mathrm{Nm}$ for 60 seconds each, after which the specimen was allowed to rest at 0 load for 60 seconds. During the data-collection cycle, load was applied quasi-statically in $1.5-\mathrm{Nm}$ increments, with each incremental load held for 45 seconds to a maximum of $6.0 \mathrm{Nm}$. Loading did not include a follower load because combined loading methods have been found to mask effects related to device stability. ${ }^{9}$

Three-dimensional specimen motion in response to the applied loads during flexibility and compression tests was determined using the Optotrak 3020 system (Northern Digital). This system stereophotogrammetrically measures the 3D displacement of infrared-emitting markers rigidly attached in a noncollinear arrangement to each vertebra. Three optical markers per vertebra were mounted on T7-10 to independently track the motion of the T7-8, T8-9, and T9-10 segments.

Custom software converted the marker coordinates to angles about each of the anatomical axes in terms of the motion segment's own coordinate system. ${ }^{6}$ Spinal angles were calculated using a technique that provides appropriate results for describing 3D spinal motion. ${ }^{8}$ From the raw load and displacement data, the mean angular range of motion (ROM), angular lax zone (LZ; portion of the ROM in which ligaments/hardware are lax), and angular stiff zone (SZ; portion of the ROM in which ligaments/hardware are under tension $)^{7}$ were quantified. These parameters are indicators of stability, with smaller values indicating greater stability. Stiffness is inversely proportional to the SZ, with a small SZ indicating high stiffness.

The mean values of the parameters from 5 conditions (intact, average short transvertebral fixation, long transvertebral fixation, average short PSR fixation, and long PSR fixation) were compared using a 1-way ANOVA followed by Holm-Šidák tests. Probability values less than 0.05 were considered significant.

\section{Results}

Images obtained from the $\mathrm{O}$-arm showed no case where 
the $50-\mathrm{mm}$ screws violated the ventral cortex of the vertebral body (Fig. 1). The mean ROM per motion segment with transvertebral screws spanning 2 levels was $66 \%$ of the mean intact ROM during flexion-extension $(\mathrm{p}=0.013)$, $69 \%$ of intact during lateral bending $(\mathrm{p}=0.015)$, and $47 \%$ of intact during axial rotation ( $\mathrm{p}<0.001)$. In comparison, the mean ROM per motion segment with PSR spanning 2 levels was $38 \%$ of the mean intact ROM during flexionextension ( $\mathrm{p}<0.001), 57 \%$ of intact during lateral bending $(\mathrm{p}=0.007)$, and $27 \%$ of intact during axial rotation $(\mathrm{p}<$ 0.001) (Fig. 4).

Adding bilateral rods across 3 levels with slightly wider transvertebral screws (i.e., TTR) resulted in a mean ROM per motion segment of $28 \%$ of the intact ROM during flexion-extension ( $\mathrm{p}<0.001), 37 \%$ of intact during lateral bending $(\mathrm{p}<0.001)$, and $30 \%$ of intact during axial rotation ( $\mathrm{p}<0.001)$. The mean ROM per motion segment for PSR spanning 3 levels was $21 \%$ of intact during flexionextension ( $\mathrm{p}<0.001$ ), 33\% of intact during lateral bending $(\mathrm{p}<0.001)$, and $22 \%$ of intact during axial rotation $(\mathrm{p}<$ 0.001) (Fig. 4).

The mean ROM per motion segment was greater with transvertebral screws and no rods than with PSR with similarly sized screws spanning 2 levels during all directions of loading, but not significantly $(\mathrm{p}>0.30)$. The mean ROM per motion segment with transvertebral screws (4.5$\mathrm{mm}$ diameter) and no rods was significantly greater than the mean ROM for the 5.0-mm-diameter TTR construct during flexion-extension $(\mathrm{p}=0.004)$ and lateral bending $(\mathrm{p}=0.027)$, but not significantly different during axial rotation $(\mathrm{p}=0.18)$. There were no significant differences in mean ROM per motion segment between the TTR construct and PSR with similarly sized screws and rods spanning 3 levels during any direction of loading $(p>0.40)$ (Fig. 4).

The mean LZ per motion segment with transvertebral screws spanning 2 levels and no rods was $42 \%$ of the mean intact LZ during flexion-extension ( $\mathrm{p}=0.032), 43 \%$ of intact during lateral bending $(\mathrm{p}<0.001)$, and $20 \%$ of intact during axial rotation $(\mathrm{p}<0.001)$. The mean $\mathrm{LZ}$ per motion segment with PSR spanning 2 levels was $24 \%$ of the mean intact LZ during flexion-extension ( $\mathrm{p}=0.013), 23 \%$ of intact during lateral bending $(\mathrm{p}=0.003)$, and $9 \%$ of intact during axial rotation $(\mathrm{p}<0.001)$.

Similarly, the mean LZ per motion segment for the TTR construct spanning 3 levels was $21 \%$ of the mean intact LZ during flexion-extension ( $\mathrm{p}=0.002), 23 \%$ of intact during lateral bending ( $\mathrm{p}<0.001)$, and $17 \%$ of intact during axial rotation $(\mathrm{p}<0.001)$. The mean $\mathrm{LZ}$ per motion segment with PSR spanning 3 levels was $11 \%$ of the mean intact LZ during flexion-extension ( $\mathrm{p}<0.001)$, $9 \%$ of intact during lateral bending ( $<<0.001)$, and $4 \%$ of intact during axial rotation $(\mathrm{p}<0.001)$.

In contrast to ROM, the mean LZ per motion segment with 4.5 -mm-diameter transvertebral screws and no rods was not significantly different than the mean LZ for TTR (5.0-mm-diameter screws) during all directions of loading $(p>0.70)$. There were no significant differences in mean LZs per motion segment between constructs with transvertebral screws and no rods and constructs with PSR spanning 2 levels during any direction of loading ( $\mathrm{p}$

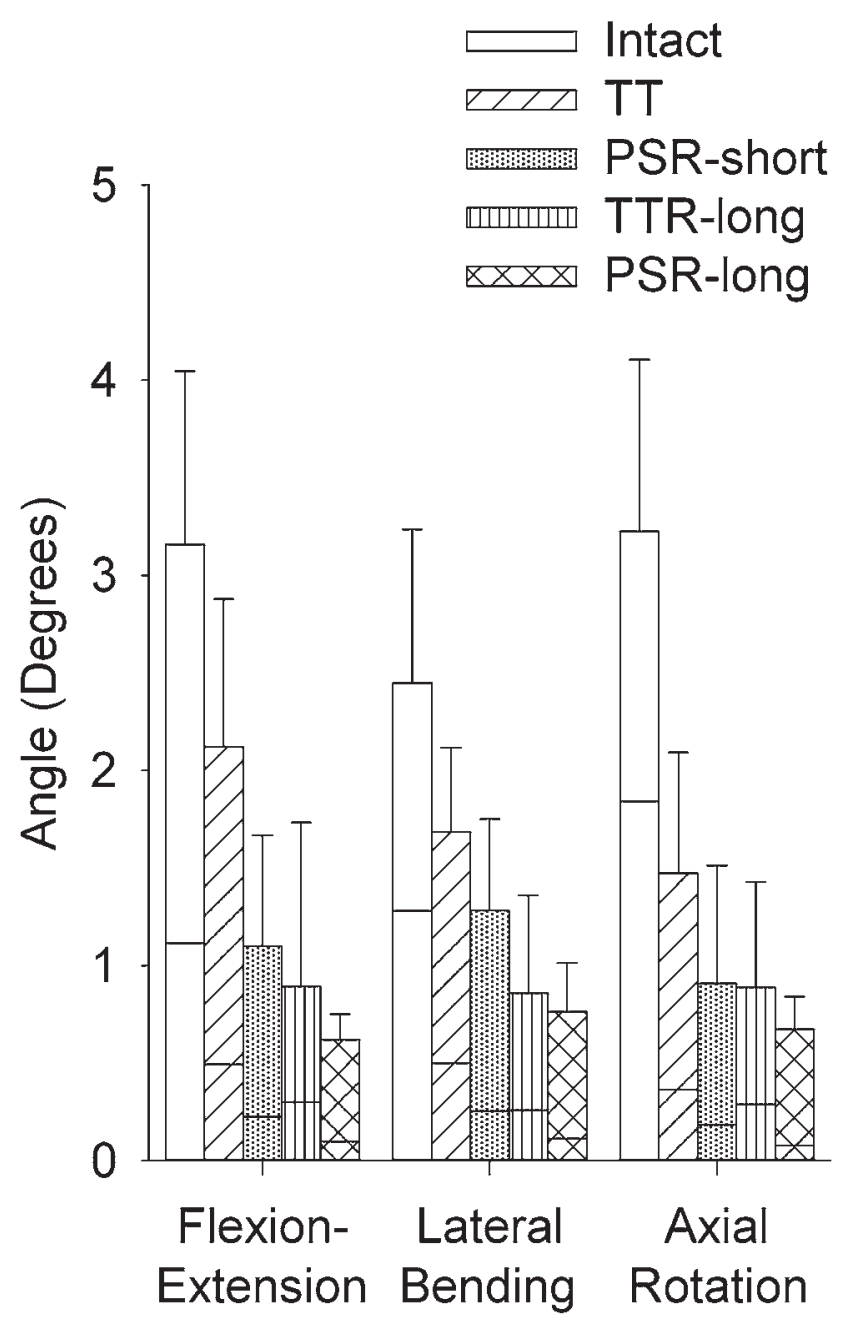

FIG. 4. The mean ROM per motion segment during flexion-extension, lateral bending, and axial rotation. Data are for the intact condition in both groups $(n=14)$. PSR-long = polyaxial pedicle screws and rods spanning 3 levels; PSR-short = polyaxial pedicle screws and rods spanning 2 levels; TT = fixed-angle transvertebral screws without rods; TTRlong = polyaxial transvertebral screws with rods spanning 3 levels. Error bars indicate $1 \mathrm{SD}$. The horizontal line in each bar indicates the division of the ROM (LZ + SZ) into LZ (portion of the ROM in which ligaments/ hardware are lax, closest to 0 angle) and SZ (portion of the ROM in which ligaments/hardware are under tension at the end of the ROM).

$>0.20$ ). Similarly, there were no statistically significant differences in the mean LZ per motion segment for TTR and PSR with similarly sized screws and rods spanning 3 levels during any direction of loading $(\mathrm{p}>0.40)$.

The mean SZ per motion segment with transvertebral screws spanning 2 levels was $78 \%$ of the mean intact SZ during flexion-extension $(p=0.285), 96 \%$ of intact during lateral bending $(\mathrm{p}=0.956)$, and $82 \%$ of intact during axial rotation $(\mathrm{p}=0.024)$. In comparison, the mean SZ with PSR spanning 2 levels was $47 \%$ of intact during flexion-extension $(\mathrm{p}=0.06), 97 \%$ of intact during lateral bending $(\mathrm{p}=0.164)$, and $51 \%$ of intact during axial rotation $(\mathrm{p}<0.001)$.

With TTR spanning 3 levels, the mean SZ per motion segment was $31 \%$ of the mean intact SZ during flexionextension ( $\mathrm{p}<0.001$ ), $50 \%$ of intact during lateral bend- 
ing (p < 0.001$)$, and $44 \%$ of intact during axial rotation (p $<0.001$ ). With PSR spanning 3 levels, the mean SZ per motion segment was $27 \%$ of intact during flexion-extension $(\mathrm{p}=0.016), 59 \%$ of intact during lateral bending $(\mathrm{p}<$ $0.001)$, and $44 \%$ of intact during axial rotation $(p<0.001)$.

The mean SZ per motion segment was significantly smaller for the construct with TTR (5.0-mm diameter) spanning 3 levels versus 4.5 -mm-diameter transvertebral screws and no rods during all directions of loading ( $p$ < 0.001). However, the mean SZ per motion segment for TTR spanning 3 levels was not significantly different than the mean SZ for PSR with similarly sized screws and rods spanning 3 levels during lateral bending $(\mathrm{p}=$ $0.875)$ or axial rotation $(\mathrm{p}=0.987)$, but was significantly greater during flexion-extension $(p=0.024)$. The mean SZ per motion segment was not different for transvertebral screws and no rods compared with the PSR construct with similarly sized screws spanning 2 levels during any direction of loading $(\mathrm{p}>0.20)$.

\section{Discussion}

In our study, transvertebral screw fixation without rods spanning 2 vertebrae in the thoracic spine significantly reduced mobility compared with intact specimens in all directions of loading, with the decrease in ROM mainly due to a decrease in LZ rather than SZ. The mean SZ remained almost unchanged during lateral bending $(96 \%$ of intact). Adding rods to the transvertebral screws increased the stability even further, with the resulting mobility equaling that with similarly sized pedicle screws and rods spanning the same length during all directions of loading.

Transvertebral pedicle screws provide the theoretical advantage of having the ability to purchase multiple cortical surfaces across 2 vertebral levels. Transvertebral screws have been used as part of a construct in the fixation of L5-S1 spondylolisthesis and have been biomechanically proven to provide stability equivalent to combined interbody/pedicle screw fixation. However, the biomechanical impact of transvertebral screws alone, when they are inserted across 2 vertebral bodies, has not been studied. Our goal was to assess the stability provided by the transvertebral screws to the segment through which they traverse.

Minamide et al. ${ }^{11}$ compared fixation with an L-5 pedicle screw and an S-1 transdiscal screw connected by a rod to fixation with the standard L5-S1 PSR construct. They demonstrated that the transdiscal construct was 1.6-1.8 times stiffer than the standard pedicle screw fixation. Additionally, the stability of the transdiscal construct was comparable to that of combined interbody/pedicle screw fixation. However, the use of transvertebral screws for stabilizing the spine at sites other than L5-S1 has not been evaluated. Their use as part of a construct in thoracic spine stabilization has recently been documented for a number of indications but primarily as a rescue for failed pedicle screw placement..$^{12}$

The morphology of the thoracic vertebrae has been studied in detail to facilitate safe insertion of pedicle screws in the thoracic spine. However, thoracic pedicle screw insertion remains a challenge in view of variations of the pedicle angle in the horizontal plane and the relatively smaller axial diameter of the pedicles, where screw insertion can result in breakage of the medial cortex, with subsequent neurological deficits.

Although the vertebral anatomy makes it more difficult to insert pedicle screws in the thoracic spine compared with the lumbar spine, the thoracic anatomy does have certain attributes that favor the insertion of transvertebral screws. First, because the sagittal pedicle angle (measured between the superior endplate of the vertebral body and a line extending down the axis of the pedicle) is greater in the thoracic spine than in the lumbar spine, a screw placed down the axis of a thoracic pedicle is angled more cephalad in relation to the superior endplate; it thus has a greater chance of being able to engage the superior endplate. In addition, because the sagittal diameter of the thoracic pedicle is typically greater than its axial diameter, a screw that is maximized to the axial diameter can be placed with a more cephalad angle across the sagittal diameter of the pedicle. Finally, the thoracic disc space height is usually shorter than the lumbar disc space height, which decreases the distance needed to capture the superior vertebral body by a screw traversing the disc space. The use of $50-\mathrm{mm}-$ long screws in the thoracic spine may be considered an aggressive technique. Although violations through the ventral cortex were not observed in the current cadaveric study, it is advisable to obtain preoperative CT scans before inserting screws to avoid any inadvertent clinical complications.

As a basic rule, any instrumentation technique should provide stability that is at least comparable to the normal spine in all directions of loading-mainly flexionextension, axial rotation, and lateral bending. In the current study, the transvertebral screw fixation technique reduced the mobility of the thoracic spine, compared with the intact status, in all directions of loading $(66 \%$ of intact during flexion-extension, $69 \%$ of intact during lateral bending, and $47 \%$ of intact during axial rotation), with the decrease in ROM mainly due to a decrease in the $\mathrm{LZ}$ rather than a decrease in the SZ. In this era of spinal instrumentation, and in view of clinical and biomechanical advantages, bilateral PSR is considered the gold-standard technique for stabilization of the spine. Hence, we compared the transvertebral technique to PSR and found that PSR spanning 2 levels provides significantly better stability during flexion-extension (38\% vs $66 \%$ of intact) and axial rotation (27\% vs $47 \%$ of intact), compared with the transvertebral technique. During lateral bending, the PSR construct provided better stability compared with the transvertebral technique (57\% vs $69 \%$ ), but this difference was not statistically significant. We also found that transvertebral (polyaxial) screws with rods spanning 3 levels provided equivalent stability to PSR fixation (using the same size polyaxial screws) spanning 3 levels. It should be noted that the aim of the study was to evaluate the overall efficacy of transvertebral screws as a possible salvage procedure in cases where thoracic pedicle screws cannot be placed. Assessment of the novel technique in a spine with a fracture, a significant multilevel spinal deformity stress, or other condition was not addressed and could be the focus of a future study. 
In summary, the insertion of 2 transvertebral screws (bilaterally at 1 level) significantly reduces the mobility of the spinal motion segment compared with the intact spine (in all directions) across that level, but it reduces the mobility significantly less than the gold-standard technique (PSR fixation with 4 screws and 2 rods), especially during flexion-extension and axial rotation. However, fixation with transvertebral screws and bilateral rods across more than 1 level is equivalent to PSR fixation in the thoracic spine.

Nottmeier and Pirris, ${ }^{12}$ in their clinical study of transvertebral screws, documented stability across 25 of 26 segments fused, resulting in good fusion rates. They have used these screws for indications including osteoporosis, sagittal deformity, and pseudarthrosis, but they primarily used transvertebral screws as a thoracic pedicle screw salvage procedure. Their study involved the use of transvertebral polyaxial screws as part of a long-segment construct connected by rods. Additionally, they used the screws in conjunction with standard pedicle screws to provide 4-point fixation at the most superior vertebral segment in long-level constructs. Consequently, their results do not directly reflect the stability provided by the transvertebral construct. However, these results emphasize the role that these screws may play in a salvage procedure or in providing 4-point fixation at the tops of a construct. Transvertebral screws may also biomechanically improve the stability of a multilevel thoracic construct, taking into account the inherent stability conferred by the transvertebral screws across the levels where they are inserted. Thus, the aim of our study was to examine the biomechanical properties of the stand-alone transvertebral construct. Future studies should be directed toward studying the pullout strength and toggle of transvertebral screws in both thoracic and lumbar spines.

\section{Conclusions}

The biomechanical results of this study indicate that the use of transvertebral screw fixation as part of a posterior fixation construct in the thoracic spine is a viable therapeutic option for fixation in a salvage procedure, if pedicle screw insertion is not possible or fails at a particular level. Further testing with a larger number of specimens is needed to determine conclusively if there is an advantage in strength with the addition of this technique. Clinical trials are also necessary to evaluate the clinical accuracy and advantages of this construct.

\section{References}

1. Abdu WA, Wilber RG, Emery SE: Pedicular transvertebral screw fixation of the lumbosacral spine in spondylolisthesis. A new technique for stabilization. Spine (Phila Pa 1976) 19:710-715, 1994

2. Aghayev K, Gonzalez-Blohm SA, Doulgeris JJ, Lee WE III, Waddell JK, Vrionis FD: Feasibility and biomechanical performance of a novel transdiscal screw system for one level in non-spondylolisthetic lumbar fusion: an in vitro investigation. Spine J 14:705-713, 2014

3. Chell J, Quinnell RC: Transvertebral pedicle fixation in se- vere grade spondylolisthesis. Report of three cases. J Neurosurg 95 (1 Suppl):105-107, 2001

4. Cinotti G, Gumina S, Ripani M, Postacchini F: Pedicle instrumentation in the thoracic spine. A morphometric and cadaveric study for placement of screws. Spine (Phila Pa 1976) 24:114-119, 1999

5. Crawford NR, Brantley AG, Dickman CA, Koeneman EJ: An apparatus for applying pure nonconstraining moments to spine segments in vitro. Spine (Phila Pa 1976) 20:20972100,1995

6. Crawford NR, Dickman CA: Construction of local vertebral coordinate systems using a digitizing probe. Technical note. Spine (Phila Pa 1976) 22:559-563, 1997

7. Crawford NR, Peles JD, Dickman CA: The spinal lax zone and neutral zone: measurement techniques and parameter comparisons. J Spinal Disord 11:416-429, 1998

8. Crawford NR, Yamaguchi GT, Dickman CA: A new technique for determining 3-D joint angles: the tilt/twist method. Clin Biomech (Bristol, Avon) 14:153-165, 1999

9. Cripton PA, Bruehlmann SB, Orr TE, Oxland TR, Nolte LP: In vitro axial preload application during spine flexibility testing: towards reduced apparatus-related artefacts. J Biomech 33:1559-1568, 2000

10. Logroscino CA, Tamburrelli FC, Scaramuzzo L, Schirò GR, Sessa S, Proietti L: Transdiscal L5-S1 screws for the treatment of adult spondylolisthesis. Eur Spine J 21 (Suppl 1):S128-S133, 2012

11. Minamide A, Akamaru T, Yoon ST, Tamaki T, Rhee JM, Hutton WC: Transdiscal L5-S1 screws for the fixation of isthmic spondylolisthesis: a biomechanical evaluation. J Spinal Disord Tech 16:144-149, 2003

12. Nottmeier EW, Pirris SM: Placement of thoracic transvertebral pedicle screws using 3D image guidance. J Neurosurg Spine 18:479-483, 2013

13. Panjabi MM: Biomechanical evaluation of spinal fixation devices: I. A conceptual framework. Spine (Phila Pa 1976) 13:1129-1134, 1988

14. Roy-Camille R, Saillant G, Mazel C: Plating of thoracic, thoracolumbar, and lumbar injuries with pedicle screw plates. Orthop Clin North Am 17:147-159, 1986

15. Xu R, Ebraheim NA, Ou Y, Yeasting RA: Anatomic considerations of pedicle screw placement in the thoracic spine. Roy-Camille technique versus open-lamina technique. Spine (Phila Pa 1976) 23:1065-1068, 1998

\section{Disclosures}

Dr. Nottmeier is a consultant for Medtronic Navigation, Globus Spine, and DePuy Synthes Spine. He also receives royalties from Globus Spine.

\section{Author Contributions}

Conception and design: Crawford, Nottmeier, Pirris, Theodore. Acquisition of data: Rodriguez-Martinez, Savardekar, Reyes, Newcomb. Analysis and interpretation of data: Crawford, Newcomb. Drafting the article: Rodriguez-Martinez, Savardekar. Critically revising the article: Crawford, Nottmeier, Pirris, Newcomb. Reviewed submitted version of manuscript: Crawford, Nottmeier, Pirris. Statistical analysis: Newcomb. Administrative/technical/ material support: Mendes, Kalb. Study supervision: Crawford, Theodore.

\section{Correspondence}

Neil R. Crawford, c/o Neuroscience Publications, Barrow Neurological Institute, St. Joseph's Hospital and Medical Center, $350 \mathrm{~W}$. Thomas Rd., Phoenix, AZ 85013.email: neuropub@dignityhealth. org. 